\title{
Combat d'un bourgeois parisien Christophe de Bordeaux et son »Beau recueil de plusieurs belles chansons spirituelles« (vers 1569-1570)
}

Les historiens des guerres de Religion ont accordé beaucoup d'importance au rôle des disputes, de la prédication ou encore des imprimés dans les luttes interconfessionnelles ${ }^{1}$. Ces modes d'affrontement étaient surtout réservés aux professionnels: prêtres, prédicateurs ou ministres. Les recherches sur les disputes religieuses montrent comment les non-spécialistes furent progressivement écartés de la participation aux discussions sur la doctrine, même si leur adhésion restait l'un des enjeux de ces affrontements ${ }^{2}$.

Et pourtant, certains laïcs souhaitaient aussi prendre part au combat pour l'honneur de Dieu, comme le bourgeois parisien Christophe de Bordeaux (vers 1537-après 1613), auteur de plusieurs recueils de chansons, de récits de miracles et d'occasionnels. Nous ne savons que peu de choses sur ce personnage. Un acte notarié de 1606 indique qu'à cette date il vivait dans la paroisse Saint-Séverin avec sa troisième femme, Ursula Rousseau, et que son fils issu du deuxième mariage, Jean de Bordeaux, était devenu imprimeur ${ }^{3}$. Christophe était donc probablement lié à la famille des imprimeurs portant le même nom ${ }^{4}$. Si nous ne connaissons rien sur son activité professionnelle, sa chanson sur le siège de Chartres par l'armée du prince de Condé affirme qu'il s'était engagé dans l'armée royale pour défendre la religion catholique et qu'il avait participé à la défense de la ville lors de cet événement. Christophe commença à écrire vers 1559 , à l'âge de vingt-deux ans. En 1569-1570, parut la somme de son œuvre poétique, le

1 Voir, p. ex., Olivier Christin, Confesser sa foi. Conflits confessionnels et identités religieuses dans l'Europe moderne ( $\mathrm{XVI}^{\mathrm{e}}-\mathrm{XVII}{ }^{\mathrm{e}}$ siècle), Paris 2009; Tatiana DeBBAgi BARANOVA, À coups de libelles. Une culture politique au temps des guerres de Religion (1562-1598), Genève 2012 (Cahiers d'humanisme et Renaissance, 104); Luc RACAuT, Hatred in Print. Catholic Propaganda and Protestant Identity During the French Wars of Religion, Aldershot 2002.

2 Jérémie FOA, Plus de Dieu l'on dispute et moins l'on en fait croire. Les conférences théologiques entre catholiques et réformés au début des guerres de Religion, dans: Piroska NAGY, Michel-Yves PERrin, Pierre RagON (dir.), Les controverses religieuses entre débats savants et mobilisations populaires, Rouen 2011, p. 79-101.

3 Archives nationales de France, Y 151, Registres d'insinuation tenus au greffe du Châtelet, fol. 725, donation de Christophe de Bourdeaux.

4 Un marchand libraire, Jean de Bourdeaulx, est installé à Paris à la fin du Xv $\mathrm{x}^{\mathrm{e}}$ siècle (acte notarié du 14 novembre 1488). Jean II de Bordeaux exerce entre 1560 et 1583 . Le fils de Christophe de Bordeaux est certainement le libraire imprimeur Jean III de Bordeaux, qui exerce au début du XVII ${ }^{\mathrm{e}}$ siècle. Voir Paul RenOUARD, Répertoire des imprimeurs parisiens. Libraires, fondeurs de caractères et caractères d'imprimerie. Depuis l'introduction de l'imprimerie à Paris (1470) jusqu'à la fin du XVI ${ }^{\mathrm{e}}$ siècle, Paris 1965, p. 46. 
»Beau recueil de plusieurs belles chansons spirituelles, avec celles des Huguenots heretiques $\&$ ennemis de Dieu, \& de nostre mère sainte Eglise « ${ }^{5}$. Publié à Paris, chez Madeleine Berthelin, ce recueil regroupe soixante-douze chansons relatives aux événements allant de 1559 à 1569. C'est un ouvrage de petit format, l'in-duodécimo, facile à glisser dans une poche de vêtement. Les caractères sont minuscules car la page comporte environ vingt-huit lignes. Les caractéristiques du recueil ne correspondent donc pas à celles que les historiens considèrent comme typiques pour les éditions dites populaires: de gros caractères et un petit nombre de pages. Il semble donc destiné au lecteur aisé, pour lequel la lecture ne présente pas de difficulté majeure, et conçu pour pouvoir accompagner partout son propriétaire. Mais au-delà de ce support éditorial qui, habituellement, reprend des chansons à succès, ce genre est avant tout oral et donc accessible à tous les publics.

Au milieu du recueil, sont insérées douze poésies du frère bénédictin Léger de Bontemps. Il serait logique de s'attendre à ce que les deux auteurs se partagent les thématiques: des chansons sur les sujets politiques pour Christophe de Bordeaux et des chansons spirituelles pour Léger de Bontemps. Or, il n'en est rien, car Christophe est l'auteur des chansons sur les points doctrinaux en controverse, malgré sa condition de laïc. Ce personnage énigmatique de bourgeois poète apparaît comme l'un des principaux auteurs parisiens qui, aux côtés des prédicateurs et des curés, proposaient à la population des analphabètes et des »demi-lettrés«, selon l'expression de Roger Chartier, des grilles de lecture des événements religieux et politiques ${ }^{6}$. Afin de comprendre quel sens Christophe de Bordeaux donnait-il à son action d'écriture, il serait intéressant de s'interroger sur ses choix thématiques et sa façon d'adapter l'information politicoreligieuse à ce genre poétique avant d'analyser la posture revendiquée par l'auteur, qui semble être celle d'un poète consolateur de ses concitoyens et frères en Christ.

Le »Beau recueil« porte l'empreinte d'une volonté d'instruire par la chanson aussi bien dans le domaine de la bonne doctrine et des pratiques dévotionnelles que dans celui, politique, de la vie de la cité et du royaume. Or, dans le feu du combat pour préserver l'autorité de l'Église, il n'était pas évident, pour un laïc, de toucher aux sujets en lien avec les querelles doctrinales. Pour légitimer sa démarche, Christophe de Bordeaux insère au milieu du recueil douze chansons de Léger de Bontemps, moine bénédictin de Saint-Bénigne de Dijon et auteur de nombreux ouvrages contre l'hérésie'. Elles affirment le rôle central de l'Église et des sacrements dans le salut et

5 Beau recueil de plusieurs belles chansons spirituelles, auec ceux des Huguenots heretiques \& ennemis de Dieu \& de nostre mere saincte Eglise. Faictes \& composees par maistre Christofle de Bourdeaux, Paris s.d, fol. 61-62v.

6 Roger ChARTIER, Lectures et lecteurs de l'Ancien Régime, Paris 1987, p.118.

7 Le catalogue »French Vernacular Books« compte vingt-trois éditions pour environ dixsept titres d'ouvrages composés par Léger de Bontemps: Andrew PeTTEGREE, French Vernacular Books. Books Published in the French Language before 1601, Leyde 2007. Ce sont surtout des ouvrages de polémique contre les protestants, comme le traité »De la Puissance et authorité du pape [...] contre ceulx du jour d'huy qui par mocquerie appellent les bons chrestiens papistes« (Paris 1565). 
l'autorité de ses docteurs, assistés du Saint-Esprit, conformément à la promesse du Christ. Léger de Bontemps utilise des mélodies composées pour les poésies amoureuses de Clément Marot par Claudin de Sermisy8. Ses chansons sont regroupées en bloc, précédées d'une adresse »Au lecteur chrétien« et suivies par un »Huictain au lecteur «?. Ce dispositif semble indiquer la reprise d'un recueil déjà imprimé. Cette insertion permet d'apporter à l'entreprise de Christophe de Bordeaux la caution spirituelle d'un homme d'Église. Au moment de la publication du »Beau recueil«, Léger de Bontemps est mort depuis déjà quatre ans. Notre auteur bénéficie donc d'une totale liberté dans l'utilisation de sa production.

Ainsi armé, non seulement il propose des chansons de dévotion à l'honneur de Marie, de Jésus Christ et des saints, mais aussi aborde des points de controverse avec les protestants. Sa »Chanson contre les Huguenots sur les articles de la foy« permet de mémoriser les différences entre le catholique et l'hérétique. Après avoir résumé l'histoire de la résurrection de Jésus Christ, il insiste sur la présence réelle du corps et du sang dans les espèces consacrées ${ }^{10}$. Il apprend à son auditoire les signes distinctifs des protestants: ils ne reconnaissent pas la confession, le purgatoire, les offices pour les trépassés, le culte marial et le culte des images. La chanson s'achève sur une note victorieuse: les hérétiques iront à la messe ou seront pendus ou brûlés »comme pourceaux«. La chanson laisse apparaître l'influence discrète des »Disputes de Guillot le porcher et de la bergère de Saint-Denis en France« d'Artus Désiré. Cet auteur introduit, en effet, la comparaison entre les hérétiques et les porcs, car Guillot vient à Genève chercher ses pourceaux pour les brûler. De plus, ce poème explique le passage de la »Chanson contre les Huguenots « qui signale comme trait distinctif d'un hérétique le fait qu'il n'ose appeler le Sauveur »Jésus«. La bergère d'Artus Désiré qui polémique avec Calvin accuse ce dernier de dire »Seigneur" au lieu de »notre Seigneur" et »Christ« au lieu de »Jésus Christ«, ce qui, d'après elle, serait un signe de la nonappartenance de Calvin et des calvinistes à la communauté des chrétiens ${ }^{11}$. Malgré cette influence, Christophe de Bordeaux apparaît comme un auteur original particulièrement doué pour l'adaptation de l'information aux lecteurs ou auditeurs peu instruits. Aucun des textes d'Artus Désiré ne récapitule de façon simple l'ensemble des différences de doctrine et de pratique entre les confessions. Léger de Bontemps avait publié, en 1560, à Lyon, une »Chanson spirituelle contre les Lutheriens \& Calvinistes, heretiques de nostre temps ${ }^{« 12}$ qui résumait les points de la controverse, mais elle était

8 P. ex., la »Premiere chanson spirituelle en la personne du ferme catholique« se chante sur l'air de »Vous perdez temps de me dire mal d'elle« et la »Chanson en la personne de l'Eglise« se chante sur »Secourez moi ma Dame par amour«.

9 Beau recueil (voir n. 5), fol. 64-78.

10 Ibid., fol. 24-25v: »son corps nous a laissé / soubs pain \& vin caché / le mistère est trop haut / pour ses fins huguenots«.

11 Arthus DÉSIRÉ, Les disputes de Guillot le porcher et de la Bergère de S. Denis en France contre Jehan Calvin prédicant de Genesve. Sur la vérité de nostre saincte foy catholicque et religion chrestienne, Paris 1559, fol. 3-4.

12 Léger de Bontemps, Chanson Spirituelle. Contre les Lutheriens \& Caluinistes, heretiques de nostre temps, Lyon 1560. 
deux fois plus longue et beaucoup plus complexe que celle de notre auteur. Léger de Bontemps cite, par exemple, les noms des docteurs de l'Église, de saint Jérôme à saint Chrysostome, dont les écrits font autorité pour les catholiques. Rien de tel chez notre chansonnier parisien: ses chansons sont courtes et claires, épurées d'allusions. Plusieurs d'entre elles sont consacrées au récit des événements emblématiques cités par les polémistes mais parfois peu connus de la population. Alors que Léger de Bontemps et Artus Désiré évoquent au passage la victoire des rois de France sur l'hérésie albigeoise pour rassurer les fidèles et prouver l'invincibilité de l'Église, Christophe de Bordeaux consacre une chanson entière à l'histoire de la lutte contre les cathares ${ }^{13}$.

Il faut peut-être chercher la raison de cette capacité d'adaptation dans le caractère très circonstanciel de cette production destinée à répondre aux besoins immédiats de la population. Si le »Beau recueil« réédite l'ensemble des chansons à succès, l'existence de quelques impressions séparées prouve qu'à l'origine la composition, la publication et peut-être la commercialisation d'une chanson intervenaient à la suite d'un événement précis. Ainsi, Christophe de Bordeaux écrivit la chanson sur l'histoire de la sainte larme en été 1562, après l'arrivée, à Paris, de cette relique de Vendôme qu'il fallait protéger des actes iconoclastes fréquents dans la région. Elle était exposée dans l'abbaye Saint-Germain-des-Prés et attirait les foules ${ }^{14}$. Le petit opuscule racontant son histoire sous une forme simplifiée et mise en musique devait certainement avoir beaucoup de succès. La création de la chanson sur le saint Roch correspond vraisemblablement à l'épidémie de peste qui sévissait dans la capitale à l'automne 1562, car ce saint était réputé pour son pouvoir de protection contre cette maladie ${ }^{15}$.

L'écriture de Christophe de Bordeaux est donc étroitement liée à la vie de la ville de Paris. Son histoire immédiate est présentée, dans le »Beau recueil«, comme celle du peuple de Dieu. Dans cette optique, la connaissance des événements du conflit religieux apparaît indispensable. À l'instar de l'histoire de l'hérésie albigeoise, elle permet de rassurer les catholiques sur le caractère indestructible de l'Église. Le bourgeois poète chante essentiellement les victoires catholiques. Ainsi, en 1562, l'événement le plus célébré est la fuite des protestants de Paris au début de la première guerre de Religion. En effet, à la mi-avril, une déclaration royale interdit le culte protestant dans la capitale et, le 26 mai 1562, le roi de Navarre, gouverneur de Paris, enjoint aux protestants de quitter Paris dans les quarante-huit heures ${ }^{16}$. Ces mesures provoquent le

13 Chansons nouvelles des bons Roys de France qui ont regné par cy-devant, soustenant la querelle de Dieu, \& de nostre mère saincte Eglise, sur le chant: Je suis fâché contre mon Capitaine, Beau recueil (voir n. 5), fol. 2-3v.

${ }^{14}$ Les miracles de nostre Sauueur Iesus Christ. Faict à la resurrection du Lazare \& de sainte Larme. Sur le chant de la Turterelle, ibid., fol. 17-18v. J. Bouillart, Histoire de l'abbaye royale de Saint-Germain-des-Prés, Paris 1724, p. 192.

15 Cette chanson fut imprimée en plaquette, sans nom d'auteur, sous le titre »La vie et légende de monseigneur saint Roch«, Paris 1562.

16 Déclaration du roy sur le faict et police de la religion portant défense de faire presches \& conveticules en la ville, faulbourg et banlieux de Paris, Paris 1562; Barbara B. DIEFENDORF, Beneath the Cross. Catholic and Huguenots in Sixteenth-Century Paris, New York, Oxford, 1991, p. 65. 
départ de la communauté protestante. Au moins huit chansons ont pour sujet la fuite des calvinistes et de leurs ministres. L'»Autre chanson qui se chante à plaisir sur le chant Te rogamus, audi nos« raille les »apostats«: Calvin, »chanoine renié«, Perrocelli qui a jeté le froc aux orties, de Bèze, souffrant de la vérole, Malo »l'essorillé« et Jean de la Rivière ${ }^{17}$. Tous, sauf Jean Calvin, ont prêché à Paris. Les chansons qui diffament les ministres autrefois redoutés pour leur pouvoir de séduction cherchent à fixer leurs noms dans la mémoire des Parisiens pour leur infliger symboliquement une punition infamante faute d'un châtiment réel. Les refrains »Et huguenots, retirez-vous, / ou vous serez pendus trestous « ou encore »ils ont perdu / dessus dessus / qui soyent pendus« affirment haut et fort qu'ils ont mérité la peine de mort. Ces chansons rappellent les scandales qui ont secoué Paris pendant les deux ans de sa »captivité«, c'est-à-dire pendant la politique de tolérance de la monarchie. Ainsi, à deux reprises, il rappelle que l'impunité des hérétiques était telle qu'ils avaient même osé présenter une requête pour demander la construction d'un temple près de Paris. Les temps ont changé car maintenant ils doivent quitter la ville ${ }^{18}$. D'autres chansons célèbrent l'exécution des hérétiques reconnus coupables de sédition, de trahison et d'assassinat: Nez d'Argent et Gabaston, acteurs de la sédition de Saint-Médard à Paris, le bailli de Pontoise, convaincu d'avoir tenté de livrer cette ville aux huguenots, Poltrot de Méré, assassin du duc François de Guise. Christophe de Bordeaux raconte les aventures macabres de leurs corps tombés entre les mains de la population parisienne, célébrant ainsi la participation de la communauté de Paris à l'exercice de la justice royale. Chanter une chanson sur le corps de l'ennemi trainé par la ville signifie participer à cette exécution et prolonger l'infamie des criminels bien au-delà de leur mort et de l'existence physique de leur corps. Dans la même logique de communion, la chanson sur la procession expiatoire du 9 juillet 1562 permet à tous d'y participer symboliquement et de demander pardon à Dieu pour l'acte iconoclaste perpétré sur la statue de la Vierge à la porte Saint-Honoré. Ces chansons délivrent donc des informations immédiatement utiles pour la communauté à la recherche de son salut. Le chant doit exprimer l'engagement du côté de Dieu et s'apparente à une œuvre pieuse. La plupart des chansons relatives à l'année 1562 ont probablement été imprimées en 1563, sous le titre »Les tenebres et regrets des predicans«, l'opuscule mentionnée par Brunet mais qui reste introuvable aujourd'hui19.

Les chansons écrites pendant les deux guerres suivantes, entre 1567 et 1570 , changent d'optique en témoignant de la politisation du conflit. Christophe de Bordeaux se met à dénoncer les protecteurs de l'hérésie, les chefs nobiliaires protestants. Le prince de Condé, l'amiral de Coligny, Gabriel de Lorges, comte de Montgomery, et François, duc de la Rochefoucauld, sont désignés comme les principaux ennemis de la commu-

17 Beau recueil (voir n. 5), fol. 3-4v.

18 Chanson nouvelle contre les adversaires de la foy catholique, qui se chante sur le chant, Nous avons un nouveau Roy en nostre pays de France et Autre chanson sur le chant, L'autre jour me acheminois mon chemin à Nanterre, Beau recueil (voir n. 5), fol. 26-27v et 31-32.

19 Jacques-Charles BRUNET, Manuel du libraire et de l'amateur de livres, Paris 1860, vol. II, p. 1111. 
nauté catholique. Les chansons relatives à cette période privilégient la célébration des victoires militaires des catholiques. On peut citer la bataille de Saint-Denis (10 novembre 1567), la défense de Chartres, à laquelle Christophe de Bordeaux affirme avoir participé (24 février-15 mars 1568), les batailles de Jazeneuil (1568), de Jarnac (13 mars 1569) et de Moncontour (3 octobre 1570). L'histoire immédiate de ces guerres est avant tout celle des victoires du peuple de Dieu guidé par le roi et la noblesse chrétienne, destinée à rassurer et à encourager les catholiques.

Le »Beau recueil« regroupe donc des chansons sur les questions doctrinales, les pratiques dévotionnelles et les victoires catholiques. Publié à la fin de 1569 ou au début de 1570 , au moment où le roi est entièrement engagé dans la guerre et où le triomphe sur les protestants parait possible, il se présente comme un manuel d'éducation civique à l'usage du bon catholique parisien et qui livre les connaissances nécessaires pour son engagement dans la lutte contre l'hérésie, connaissances présentées sous une forme très accessible.

En effet, le principal apport de Christophe de Bordeaux consiste dans l'adaptation de l'information politico-religieuse. Au moins cinq chansons du recueil ont été identifiées avec certitude comme composées à partir de libelles imprimés. Tel est le cas de la première pièce du recueil, »Chanson nouvelle des bons Roys de France qui ont régné par cy-devant«. Elle raconte la lutte de Philippe Auguste et de saint Louis contre les cathares en traduisant en forme poétique et orale »L'histoire des schismes et hérésies des Albigeois, conforme à celle du présent $\ll^{20}$. De même, la chanson sur la relique de la sainte larme de Jésus Christ a été composée à partir d'un texte anonyme déjà versifié, datant du début du siècle, »Le discours comme la saincte Larme fut apportée en l'abbaye de Vendosme par le noble Comte Geoffroy Martel $\ll^{21}$. Cette plaquette était destinée, à l'origine, aux pratiques dévotionnelles des pèlerins, car elle comportait des extraits de l'office en latin. Rééditée à Paris à l'occasion de l'exposition de la relique en $1562^{22}$, la poésie fut reprise par Christophe de Bordeaux qui raccourcit l'histoire, simplifia sa trame événementielle et la mit en musique ${ }^{23}$. Les chansons sur les dernières paroles du duc François de Guise, sur la procession parisienne lors de ses funérailles ou encore sur la bataille de Moncontour furent également composées à partir des relations imprimées. La mise en chanson de libelles permet aux informations de passer de la sphère de l'écrit à la sphère de l'oral et les rend accessibles à l'ensemble de la population.

20 J. GAY, L'histoire des schismes et hérésies des Albigeois, conforme à celle de présent, Paris 1561.

21 S'ensuit le Mistère de la saincte Larme, comment elle fut apporté de Constantinople à Vendosme, s.l.n.d, Anatole de Montaiglon (éd.), Recueil de poésies françoises des $X^{\mathrm{e}}$ et $\mathrm{XVI}^{\mathrm{e}}$ siècles, Paris 1855 , vol. I, p. 43-54.

22 Le discours comme la saincte Larme fut apportée en l'abbaye de Vendosme par le noble Comte Geoffroy Martel, avec les miracles, oraisons et messe de ladicte saincte Larme, Paris 1562, Bibliothèque nationale de France (Richelieu), ms. lat. 12700, fol. 306-318.

${ }^{23}$ Les miracles de nostre Sauueur Iesus Christ (voir n. 14), fol. 17-18v. 
Cette proximité avec les imprimés permet d'analyser le travail d'adaptation du texte. $\mathrm{La} » C h a n s o n$ nouvelle de madame de Guise et la remonstrance que luy faict monsieur son mary« est composée à partir du libelle »Recueil des derniers propos que dit et tient feu tresillustre Prince, Messire Françoys de Lorraine, duc de Guyse«. Les deux textes mettent en scène la mort exemplaire du duc, champion de la cause catholique, mortellement blessé par un gentilhomme protestant, Jean Poltrot de Méré, le 18 février 1563. Leur parenté ne fait pas de doute. Voici comment François de Guise s'adresse à son fils aîné, Henri:

\section{CHANSON NOUVELLE}

Et mon filz, ici présent,

Le plus avancé en âge,

Sois au Roy obéissant

Luy rendent foy \& hommage.

Or, mon filz, as-tu ouy

Ce que $\mathrm{j}$ 'ay dict à ta mère,

Que Dieu te laisse aujourd'huy

Pour te regir comme père.

Il te la fault honorer

Et luy rendre obéissance

Et sainctement reverer

Car c'est de Dieu l'ordonnance.

Aye mon filz, mon amy,

L'amour de Dieu \& la crainte

Et de ton pervers ennemy

La force sera estainte 24 .

\section{RECUEIL DES DERNIERS PROPOS}

[Le duc recommande ses enfants à son épouse]: Je vous prie de tout mon cœur les avoir tous pour recommandez, et principallement mon fils icy présent. Qui estant le plus avancé d'aage pourra servir de guyde \& d'exemple aux autres [...] Mon filz, tu as ouy ce que j'ai dit à ta mere, que Dieu te laisse pour tenir ma place. Je te commande de luy estre obéissant, \& de luy rendre tousjours honneur \& reverance, suyvant les bons conseilz \& prudentes instructions qu'elle te donnera. Aye mon mignon, mon amy, l'amour \& la crainte de Dieu, principalement devant tes yeulx \& dedans ton cœur, chemine selon ses voyes [suivent des conseils pour éviter les erreurs de jeunesse]. Employe entierement ton pouvoir, \& ta vie, pour t'en acquiter selon ton devoir, à l'honneur de Dieu \& au contentement de ton Roy: lequel tu doibs recongnoistre apres Dieu, pour souverain maistre $\&$ seigneur, du tout luy dediant tes services 25 .

La proximité de plusieurs expressions et tournures de phrase montre que Christophe de Bordeaux a traduit le libelle en chanson avec un véritable souci de rester fidèle aux paroles du duc. Mais les aménagements effectués sont très importants. Il opère de nombreuses coupes pour alléger le texte. Il enlève des paroles que François de Guise adresse à ses frères cardinaux et à l'assistance, aussi bien que des passages sur l'état déplorable de la France. Il ne garde que des recommandations laissées à son épouse et à son fils ainé et l'oraison finale par laquelle le mourant remet son âme entre les mains de Dieu. Christophe de Bordeaux omet également des passages qui risquent d'atténuer la perfection du duc. Dans l'imprimé, celui-ci demande pardon à son épouse pour les offenses passées, quoiqu'il ne se tienne pas »des plus grands pécheurs en cest endroit«. Il l'exhorte, ensuite, devant Dieu, à s'appliquer à la bonne éducation de leurs enfants et prie le Seigneur de la punir si elle devait oublier ses recommandations et se rendre trop

${ }^{24}$ Chanson nouvelle de madame de Guyse, et de la remonstrance que luy faict monsieur son mary, sur le chant. Delaissez la verde couleur, ibid., fol. 10-11.

25 Recueil des derniers propos que dit et teint feu tresillustre Prince, Messire Françoys de Lorraine, duc de Guyse prononcez par luy peu devant son trespas a Madame la Duchesse sa femme, Monsieur son filz, Messieurs les Cardinaulx ses freres, \& a plusieurs assistans a l'heure de son trespas, Paris 1563, fol. 3-5v. 
sévère ou trop nonchalante à leur égard ${ }^{26}$. Notre auteur évite ces allusions aux fautes passées ou possibles dans l'avenir car elles nuiraient au caractère idyllique de la scène. En revanche, il introduit un élément narratif et fictionnel. Alors que l'imprimé commence directement par les paroles de François de Guise, Christophe de Bordeaux débute la chanson par la description de l'épouse éplorée au chevet de son mari, main dans la main, le priant de lui confier sa pensée. Cette scène est appelée à émouvoir le chanteur et son auditoire, d'autant plus que la chanson se chante sur un air très connu et très apprécié à l'époque, »Laissez la verde couleur«, qui a été composé pour une poésie de Mellin de Saint-Gelais décrivant la mort d'Adonis.

La narration des libelles est donc romancée, débarrassée des détails jugés superflus par rapport à l'objectif revendiqué du chansonnier, celui de la consolation des chrétiens. Ainsi, la chanson sur la bataille de Moncontour rejette le récit des manœuvres des armées avant la bataille, celui de multiples revirements de l'action militaire et la liste des tués et des blessés pour mettre en avant l'élan des troupes inspirées de Dieu, sous la conduite de la noblesse chrétienne, un élan auquel rien ni personne ne saurait résister ${ }^{27}$. La plupart des chansons s'achèvent par un appel à la prière collective. Christophe de Bordeaux cherche à dégager dans l'événement son »essence« chrétienne et à l'agrémenter par une éthique et une esthétique chevaleresques. Soldat lui-même à un moment de sa vie, il est très sensible aux valeurs guerrières. Ainsi, si le courage du peuple chrétien - des soldats ou des habitants de la ville - est toujours souligné, les nobles catholiques se présentent sous les traits de preux chevaliers. Christophe de Bordeaux appréciait la chanson de Roland à laquelle il fait référence dans une épitaphe de Jean de Hans. Selon lui, la vaillance de ce prédicateur parisien qui combattait l'hérésie avec des armes spirituelles dépassait celle du »preux Roland«28. Il utilise beaucoup les airs et la métrique des chansons sur les campagnes italiennes de François $\mathrm{I}^{\text {er }}$ et d'Henri II, comme c'est le cas de »Quand Bourbon vit Marseille « ${ }^{29}$ ou de »Les Bourguignons ont mis le camp devant la ville de Péronne«. Christophe de Bor-

$26 »$ Et s'il advient que vous vous oubliez en ce dont je vous prie, vous rendant trop rigoreuse ou nonchalante en vostre debvoir envers eux, Je prie mon dieu qu'il vous en donne une forte punition pour vous faire recongnoistre vostre faulte. Je ne dictz pas cecy, mamye, pour aucune deffiance que j'ay de vous [...] Mais l'amour paternelle \& le grand desir que j'ay que vous suyviez ma volunté me faict parler en ceste sorte«, ibid., fol. $3 \mathrm{v}-4$.

27 Discours de la bataille du lundy troisieme iour d'Octobre 1569, en laquelle il a pleu à Dieu donner tres memorable victoire au Roy treschrestien par la bonne, heureuse, et vertueuse conduicte de monseigneur Duc d'Anjou son frere, \& Lieutenant general, Paris 1569; Beau recueil (voir n. 5), fol. 75-77v.

28 Christophe DE BordeAuX, Déploration sur la mort et trespas de deffunct de bonne mémoire frère Iehan de Ham, Religieulx de l'ordre des Minimes. Qui en son viuant a tousiours repulsez les faulces opinions, qu'auoient les faulx Prophetes \& ministres de l'Antechrist, contre les Sacremens de l'Eglise, pensant la ruiner, Paris 1562.

29 Pour le texte de la chanson, voir: LE ROUX DE LINCY (éd.), Recueil de chants historiques français, $2^{\mathrm{e}}$ série, Paris 1842, p. 96. Christophe de Bordeaux a mis sur cet air la »Chanson nouvelle de la deffaite de l'armée des Huguenots, rebelles \& seditieux, par Monseigneur le Duc d'Anjou frere du Roy \& les Princes Catholiques« et » $\mathrm{O}$, la folle entreprise du prince de Condé«, Beau recueil (voir n. 5), fol. 75-78 et 61-64. 
deaux puise donc son inspiration non seulement dans la production récente mais aussi dans sa connaissance de la tradition des chansons de guerre.

Notre bourgeois chansonnier se revendique, en effet, comme un poète de la cité combattante, poète qui prend en charge l'ensemble de ses aspirations spirituelles et temporelles. À la fin de nombreuses chansons, il se présente comme »un jeune garçon, enfant de cette ville«. La cité est donc sa référence de légitimation. Comme Artus Désiré, il fait partie de ces écrivains qui n'ont pas besoin, pour écrire, de l'appui d'un grand. Il recherche plutôt un succès commercial et symbolique auprès du public parisien. Son écriture échappe à la logique du jeu nobiliaire qui détermine, à cette époque, le discours d'un bon nombre de libelles. Mais Artus Désiré s'adresse à la communauté de la hauteur de sa position de prêtre, voire de prophète. Christophe de Bordeaux interpelle ses concitoyens en tant que l'un des leurs; il partage leurs joies et leurs craintes et prétend traduire leur sagesse et leur voix morale. Ce type de posture se rencontre chez quelques poètes mineurs de l'époque, comme, par exemple, Thomas Gay, poète protestant de $\mathrm{Die}^{30}$. Néanmoins, Christophe de Bordeaux prétend instruire et consoler. Il semble, en effet, fasciné par le modèle de prédicateur, comme le montre son épitaphe du minime Jean de Hans. Il l'admire pour le courage avec lequel de Hans dénonce des péchés sans égard pour les conditions des pécheurs ${ }^{31}$. Jean de Hans était, en effet, considéré par les autorités comme un prêtre séditieux. Il fut arrêté dans la nuit du 9 au 10 décembre 1561 à la suite des propos violents prononcés lors de son sermon dans l'église de Saint-Barthélemy. Cette arrestation mit Paris au bord de l'émeute. L'Hôtel de Ville délégua alors un groupe de marchands notables pour supplier le roi de le libérer. Le roi céda et, trois jours plus tard, Jean de Hans fut libéré et fit un retour triomphal dans son église. Le lendemain, une procession fut organisée pour remercier Dieu de ce signe de sa faveur. La foule des gens venus écouter son sermon fut si grande qu'elle ne pût entrer dans l'église ${ }^{32}$. Christophe de Bordeaux faisait partie de ses admirateurs. Rien n'indique qu'il s'était lui-même opposé à la politique de tolérance pratiquée par la monarchie. Dans ses chansons, publiées pendant les périodes de conflit ouvert entre les protestants et le pouvoir royal, le roi bénéficie de l'image traditionnelle de protecteur de l'Église. Mais la violence de ses appels à l'extermination physique des hérétiques et l'aspiration à la pureté absolue de la communauté devant Dieu contribuait certainement à nourrir le mécontentement des Parisiens pendant les périodes de paix.

Néanmoins, Christophe de Bordeaux revendique surtout son désir de consoler et d'inspirer la communauté, à l'image de Jean de Hans, dont les prédications apportaient

30 Mémoires des frères Gay, de Die, pour servir à l'histoire des guerres de religion en Dauphiné et spécialement dans le Diois, éd. par Jules CHEVALIER, Montbéliard 1888.

31 Chanson nouvelle de l'ymage Nostre Dame qui a esté remise à la porte sainct Honoré, ibid., fol. $5-8 \mathrm{v}$.

32 Voir Alphonse DE RuBle, L'arrestation de Jean de Hans et le tumulte de Saint-Médard, dans: Bulletin de la Société de l'histoire de Paris et de l'Ile-de-France 13 (1886), p. 84-94. 
un grand réconfort aux $»$ bourgeois et marchands $\aleph^{33}$. Cet objectif de la consolation spirituelle semble central dans le »Beau recueil« qui répète inlassablement que l'Église catholique est invincible. Le refrain d'une chanson appelle les chrétiens à abandonner leur chagrin: »Or, laissons, laissons François, triste envie et desplaisance«. Seules deux chansons sur soixante-douze appellent les pécheurs à se repentir et à se corriger et la thématique de la menace prophétique est complètement absente. Le poète adopte une posture contraire à celle, prophétique, d'Artus Désiré. Plutôt que de menacer ses concitoyens de châtiments terribles, il insère dans le recueil une "Chanson tresconsolative sur l'amour que Dieu nous porte«, qui promet le bonheur et la vie paisible à celui qui respecte les préceptes du Christ. Notre poète met dans la bouche de ceux qui chantent ses chansons, parfois très violentes, un message d'espoir. Sa violence est une sorte de violence joyeuse, terrible mais thérapeutique, qui sert à la purification et au désangoissement de la communauté. Christophe de Bordeaux s'attribue donc une mission de consolation, d'instruction et d'explication, des fonctions qui s'apparentent à ceux d'un pasteur. Il semble qu'il se plaise à jouer le rôle d'auxiliaire laïc du clergé. Il serait intéressant de savoir si cette posture d'auteur coöncidait avec une responsabilité quelconque au sein d'une confrérie ou d'une fabrique. Mais, pour l'instant, les sources restent muettes.

Le »Beau recueil« de Christophe de Bordeaux connut un vif succès. Il fut réédité chez Madeleine Berthelin deux mois après la première édition, avec quelques petites modifications ${ }^{34}$. Signe d'un succès durable, ses chansons spirituelles furent rééditées tout au long du siècle dans des recueils collectifs. On les trouve encore reproduites dans le premier quart du $\mathrm{XVII}^{\mathrm{e}}$ siècle alors que la lutte contre les calvinistes reste d'actualité. Pour sa part, Christophe de Bordeaux continua de composer et de publier des chansons et des occasionnels jusqu'en $1613^{35}$. L'activité de cet auteur prolixe mais encore peu connu laisse apparaître l'influence exercée par des laïcs dans la vie spirituelle de la communauté. De plus, sa production nous fournit une rare occasion de saisir le fonctionnement de la chanson comme un chainon manquant entre la production écrite et la culture orale et non savante des populations. Il faudrait poursuivre ce travail en s'interrogeant notamment sur les mélodies utilisées, qui ont joué un grand rôle dans le succès de ces chansons et servi de support à la mémorisation des informations politiques. De nombreux airs ont été transcrits et sont parvenus jusqu'à nous. Ainsi la chanson »O la folle entreprise du prince de Condé«, sur le siège de Chartres,

33 »C'estoit le reconfort, d'Orphelins \& de Veuves, De bourgeois \& marchans qui maintenant le pleurent«, BORDEAUX, Déploration (voir n. 28), fol. 2.

34 Recueil de plusieurs belles chansons spirituelles, faictes \& composees contre les rebelles \& perturbateurs du repos \& tranquillité de ce Royaume de France, auec plusieurs autres chansons des victoires qu'il a plu a Dieu de donner à nostre treschrestien Roy Charles IX de ce nom, Paris s.d.

35 J'ai récemment découvert un autre recueil de Christophe de Bordeaux, conservé à la bibliothèque de Bâle, »Le recueil des chansons des batailles et guerres aduenues au Royaume de France, durant les troubles« (Paris 1575), qui s'ajoute à huit titres signalés par le catalogue de »French Vernacular Books«. 
composée sur l'air »O que dit-on en France de monsieur de Bourbon«, connut un succès si important que, selon une hypothèse, elle aurait été entendue par les soldats flamands lors de la troisième guerre de Religion. Ils auraient repris, à leur tour, la mélodie, qui aurait servi de support pour »Wilhelmus«, l'hymne national hollandais. Un ensemble de musique ancienne, Camerata Trajectina, a reconstitué et enregistré cette chanson de Christophe de Bordeaux en 198436. Cette reconstitution permet de mieux se rendre compte de la puissance performative de la chanson et de toucher à cette oralité perdue des guerres de Religion.

\section{RÉSUMÉ}

Cet article examine un recueil de chansons contre les protestants composées par un bourgeois parisien, Christophe de Bordeaux (1537-après 1613). Cet auteur peu connu aujourd'hui paraît avoir joué un important rôle d'informateur et de médiateur culturel à Paris pendant les guerres de Religion. Son »Beau recueil de plusieurs belles chansons spirituelles« (1569-1570) aborde des sujets variés: les différences théologiques et liturgiques entre les catholiques et les protestants, l'expulsion des ministres protestants de Paris, les exécutions des rebelles, les processions religieuses et les victoires obtenues par les catholiques. La plupart de ces chansons avaient déjà été publiées auparavant, en plaquettes séparées, afin de proposer aux Parisiens l'interprétation civique et religieuse des événements les plus importants pour les réconforter et leur permettre de prendre part, à travers le chant, à la vie de la cité. Les chansons de Christophe de Bordeaux étaient capables de rassembler la communauté tout entière mais aussi d'atteindre le public de »demi-lettrés« (Roger Chartier) et d'illettrés, notamment grâce à la traduction en chanson de libelles et de pamphlets imprimés. Sa production fournit ainsi aux historiens une rare occasion de reconstruire un chaînon manquant entre la production écrite et la culture orale et non savante des populations. Le recueil de ce bourgeois poète se présentait comme une sorte de manuel à l'adresse des Parisiens zélés et leur fournissait des connaissances et des outils indispensables pour faire corps avec la cité catholique.

\section{SUMMARY}

This article examines a songbook against Protestants composed by a citizen of Paris, Christophe de Bordeaux (1537-after 1613). Though he has become an obscure figure, he played an important role as a news broker and a cultural mediator in Paris during the Wars of Religion. His »Beau recueil de plusieurs belles chansons « (1569-1570) deals with several topics, namely theological and liturgical differences between Catholics and Protestants, the expulsion of the Calvinist ministers from Paris, executions of protestant rebels, descriptions of religious processions and Catholic victories. Most of these songs were first published separately in reaction to a specific event. They provided the Parisians with an interpretation of the news, comforted them and induced them to take part in civic life, mainly by singing. The songs of Christophe de Bordeaux were able to move the whole community and to reach a »semi-literate« (Roger Chartier) or an illiterate audience. To make his political information more accessible, he was turning his libels and pamphlets into songs. His work gives the historians a rare opportunity to study the song as a missing link be-

36 Geuzenliederen Rond Willem Van Oranje, NKV 8403, 1984. 
tween print and oral culture. It seems that his songbook has been designed as a guideline for zealous Catholics in order to rally the civic body of Paris around relevant pieces of news. 